\title{
Mammografiescreening
}

\section{Mehr Schaden als Nutzen?}

\section{Der Nutzen der Früherkennungs- Reihenuntersuchung ist nach wie vor nicht so gut belegt, wie das manche Werbekampagnen glauben machen, schrieben wir in unserem Report in der MMW Nr.47/2010, S.14. Dazu er- reichte uns folgender Leserbrief:}

— In der Tabelle 1 „Entscheidungshilfen Mammografie" wird der Nutzen und das Risiko des Mammografiescreenings gelistet. Aber die Zeit, die für die Durchführung aufgebracht werden muss, wird nicht genannt. Auch die Kosten nicht, die ja irgendwie gegenfinanziert werden müssen.

Eine Vergleichsstudie zum Mammografiescreening aus Norwegen ergab, dass 2500 Frauen über einen Zeitraum von zehn Jahren gescreent werden müssen, um einen Todesfall zu verhindern. Ohne Screening würden $90,2 \%$ der Frauen zehn Jahre überleben, mit Screening 90,25\%. Das mache im Mittel einen gewonnenen Extratag in zehn Jahren pro Frau aus.
Alleine aber um die Screeningtermine wahrzunehmen muss eine Frau in zehn Jahren etwa einen ganzen Tag Zeit aufwenden, wenn man auch die Wege- und Wartezeiten mitrechnet. Radiologen und ihre Helferinnen, die das Screening durchführen, wenden pro gescreenter Frau in zehn Jahren

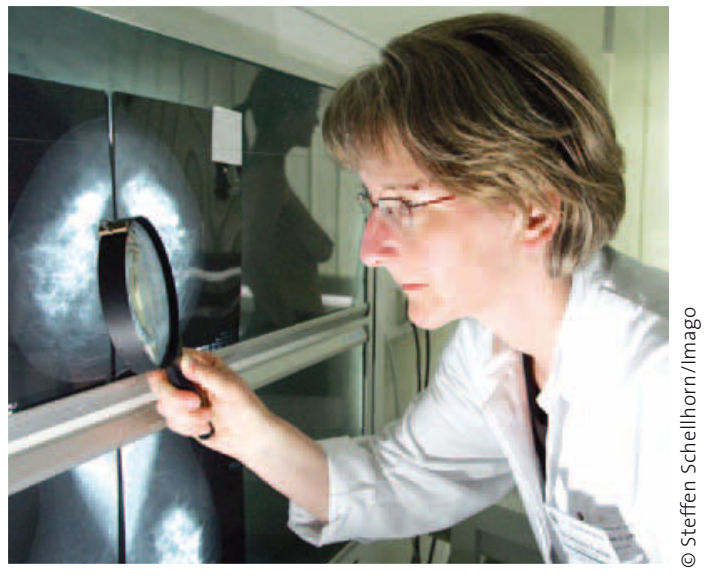

Mammografie: Lebenszeit vertan statt gewonnen? etwa einen achtel bis einen viertel Tag Zeit auf, um die Frau einzubestellen, zu röntgen, Berichte zu schreiben, alles zu organisieren usw. Und die Menschen, die arbeiten, um über ihre Beiträge oder Steuern die Kosten des Programms zu tragen, müssen dafür pro gescreenter Frau in zehn Jahren etwa acht bis zehn Stunden arbeiten. Dazu kommt der Zeitaufwand, um die unnötigen Kontrolloperationen (Gewebeentnahmen, Amputationen) bei den falsch diagnostizierten Frauen durchzuführen und gegenzufinanzieren. Dem gewonnen Extratag stehen zwei verlorene Tage gegenüber.
Bilanz: Der Nutzen ist offensichtlich geringer als der Schaden. Ähnliche Zeitbilanzen kann man auch für das PSA-Screening aufstellen. Ich finde, man sollte diese Programme nicht mehr betreiben. Außer Spesen nichts gewesen. Natürlich gibt es aber trotzdem Leute, die auf jeden Fall immer davon profitieren: Die Ärzte, die Helferinnen, die Industrie und die Verwaltung. Dorthin fließt das ganze Geld.

\footnotetext{
- Dr. med. Dieter Wettig, Facharzt für Allgemeinmedizin, Erlkönigweg 8 , D-65199 Wiesbaden-Dotzheim
}

\section{Was soll hier physikalische Therapie?}

In unserer Serie Naturheilkunde beschrieben wir in MMW Nr. 45/2010, S. 26, wie das Hämorrhoidalleiden naturheilkundlich behandelt wird. Ein Leser kritisiert:

Frau Prof. Kraft unterscheidet nicht sauber zwischen Hämorrhoiden und dem Hämorrhoidalleiden. Anamnestisch sollte nach Beschwerden im Analbereich und nicht nach Diagnosen gefragt werden.

Unstrittig ist, dass die von Frau Kraft aufgezählten Basismaßnahmen, wie z. B. eine ballaststoffreiche Kost, hilfreich sind zur Prävention und Therapie des Hämorrhoidalleidens. Wenn Hämorrhoiden symptomatisch werden, gibt es hervorragende Möglichkeiten der kausalen Therapie. Der Sinn der vorgeschlagenen physikalischen Therapie - neben Sitzbädern unterschiedlicher Temperaturen und Dauer, Reinigung mit kaltem Wasser sechsmal täglich drei Minuten (18 Minuten insgesamt also), Bewegungstherapie, Bauchlage (Oberkörper tiefer als das Becken) und Krankengymnastik - ist mir nicht ersichtlich.
Des Weiteren wird die Anwendung verschiedener Externa wie z. B. Eichenrinde, Hamamelisblätter und -rinde usw. vorgeschlagen, und das, obwohl sich der Hämorrhoidalkomplex im Bereich des distalen Rektums/proximalen Analkanals befindet. Dieser entzieht sich schon deshalb einer Behandlung analog der von Venen der unteren Extremität. Dringend abzuraten ist auch von dem Vorschlag, feuchte Reinigungstücher $z u$ verwenden. Sie enthalten $u$. a. einen Mix aus Duftstoffen, die bekanntermaßen starke Allergene sind.

Das Hämorrhoidalleiden sollte stadiengerecht kausal therapiert werden. Bewährt haben sich z. B. die Sklerosierungsbehandlung (in Deutschland in der Regel nach Blond), die Ligaturbehandlung - und bei konservativ nicht beherrschbarem oder fortgeschrittenem Befund - diverse operative Methoden.

- Dr. med. Horst Mlitz, Facharzt für Allgemeinmedizin Koloproktologie, Sulzbachstraße 20, D-66111 Saarbrücken 